\title{
Poincare invariance in temporal gauge canonical quantization and $\theta$-vacua
}

\author{
Hisashi Kikuchi \\ Department of Physics \\ University of California, Riverside \\ Riverside, CA 92521 \\ February, 1993. UCRHEP-T104
}

\begin{abstract}
The Poincare invariance in the temporal gauge canonical quantization of QCD is shown manifestly by verifying the energy-momentumvector and angular-momentum-tensor satisfy the Poincare algebra in the physical Hilbert space. Two different values of $\theta$ for the $\theta$-term in QCD lagrangian lead to different representations of the Poincare group, which are, however, connected by an unitary transformation. Thus the parameter $\theta$ becomes physically irrelevant unless we can further restrict the physical Hilbert space.
\end{abstract}




\section{Introduction}

The speculation that QCD would have multiple vacua is based on a semiclassical consideration in terms of Euclidean functional integral. The instanton solutions are interpreted as the quantum tunneling between topologically distinct $n$-vacua and the true vacuum is expected to be an appropriate linear

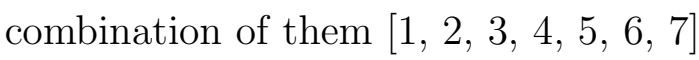

$$
|\theta\rangle \sim \sum_{n} e^{i n \theta}|n\rangle
$$

As a result we obtain a set of vacua parametrized by a real parameter $\theta$. The world of hadrons is regarded as being constructed on one of them.

The temporal gauge $\left(G_{0}^{a}=0\right)$ have been exclusively used when this semiclassical speculation is embodied in terms of the canonical quantization. This gauge fixing condition does not suffer from Gribov's ambiguity and is the most convenient one for incorporating properly large field fluctuations like instantons [8, 9]. In this gauge, however, the Lorentz invariance or, more generally, the Poincare invariance of the theory is not manifest. For the multiple vacua to be acceptable theoretically, there must be a representation of Poincare group in each $\theta$-sector, the Hilbert space constructed based on each $\theta$-vacuum. In this paper, we will verify the Poincare invariance of the temporal gauge quantization and discuss about the multiple vacua speculation from the canonical quantization view point.

The standard way to take into account the effect of the $\theta$-vacuum is to add the Pontryagin density to the lagrangian $\llbracket$. We will canonically quantize the lagrangian with the $\theta$-term and show the Poincare invariance within the physical space, the subspace of the Hilbert space specified by the requirement of gauge invariance.1 The important observation that will be shown in this paper is that different values of $\theta$ in the lagrangian do not automatically

\footnotetext{
${ }^{1} \mathrm{~A}$ similar investigation in the axial gauge have been done in Ref. [10].
} 
lead us to different $\theta$-sectors. Instead, they result in the different representations of the Poincare group in the physical space. These representations are connected by certain unitary transformations. Thus, all different values of $\theta$ make the same physical predictions. In order to confirm the semiclassical speculation on the multiple vacua and examine the $\theta$-dependence of QCD, it is important to explicitly write "large" gauge transformations in terms of field operators.

The Poincare invariance verified in this paper also gives a firm theoretical basis to a recently formulated Lorentz invariant sum rule for the $\theta$ dependence, the $\theta$-dependence of the vacuum energy in terms of a sum over matrix elements of a field operator [11].

We will ignore quark contribution and consider pure gluonic QCD. This paper is organized as follows. The section 2 contains a review of the quantization method and description of various notations. Strictly speaking, the gauge we will use is not the usual temporal gauge. We will not set the temporal components $G_{0}^{a}$ of gluon fields to be zero but fix them as arbitrary given c-number functions. We use the term "temporal gauge" in this more general sense. The advantage of keeping $G_{0}^{a}$ nonzero is that equations are written in more Poincare covariant fashion than setting $G_{0}^{a}=0$. In section 3, we explicitly verify that the energy-momentum-vector and angular-momentum-tensor obey the Poincare algebra when restricted in the physical space. At this point we assume the existence of at least one vacuum state that is invariant under spatial translation and rotation. Section 4 is devoted for discussion. The effect of $G_{0}^{a}$ and $\theta$ on physical observables will be discussed.

\section{Yang-Mills equations}

The familiar problem in quantizing gauge theory in the canonical hamiltonian formalism is the absence of momenta conjugate to the temporal components $G_{0}^{a}$. Then we cannot determine the time evolution of $G_{0}^{a}$ by the Heisenberg 
equations. The way we adopt in order to circumvent this problem is simple; we take $G_{0}^{a}$ as given c-number functions of space-time coordinates $x$ and check the independence of physical observables from $G_{0}^{a}$ at the end of the quantization procedure. Since we fix $G_{0}^{a}$, we no longer have the freedom of the time dependent gauge transformation. The canonical equations of motion for the spatial components $G_{i}^{a}$ and their conjugate momenta $\pi_{i}^{a}$ form now a closed set in the sense that the first order differential equations completely determine $G_{i}^{a}(x)$ and $\pi_{i}^{a}(x)$ for all $x^{0}$ from any given initial configuration at $x^{0}=0$.

The QCD lagrangian with the topological $\theta$-term reads

$$
\mathcal{L}=-\frac{1}{4} G_{\mu \nu}^{a} G^{a \mu \nu}+\theta \frac{g^{2}}{32 \pi^{2}} G_{\mu \nu}^{a} \tilde{G}^{a \mu \nu},
$$

where

$$
G_{\mu \nu}^{a}=\partial_{\mu} G_{\nu}^{a}-\partial_{\nu} G_{\mu}^{a}+g f^{a b c} G_{\mu}^{b} G_{\nu}^{c}, \quad \tilde{G}^{a \mu \nu}=\frac{1}{2} \epsilon^{\mu \nu \lambda \sigma} G_{\lambda \sigma}^{a},
$$

and $g$ is the coupling constant. Following the usual procedure to get the canonical form of the theory, we calculate conjugate momenta

$$
\pi_{i}^{a} \equiv \frac{\partial \mathcal{L}}{\partial \dot{G}_{i}^{a}}=\dot{G}_{i}^{a}-\mathcal{D}_{i} G_{0}^{a}-\bar{\theta} B_{i}^{a},
$$

where $B_{i}^{a}$ stand for chromo-magnetic fields,

$$
B_{i}^{a}=\frac{1}{2} \epsilon_{i j k} G_{j k}^{a},
$$

$\mathcal{D}_{\mu}$ the covariant derivative for adjoint representation, for example,

$$
\mathcal{D}_{\mu} A^{a} \equiv \partial_{\mu} A^{a}+g f^{a b c} G_{\mu}^{b} A^{c},
$$

\footnotetext{
${ }^{2}$ Notations: The Minkowski indices are denoted by Greek letters. Their lowering and raising is done by the metric $g^{\mu \nu}=g_{\mu \nu}=\operatorname{diag}(1,-1,-1,-1) . \epsilon_{\mu \nu \lambda \sigma}$ is the four dimensional completely antisymmetric tensor; $\epsilon_{0123}=-\epsilon^{0123}=1$. Roman letters $i, j, k \ldots$ are for spatial indices and run from 1 to 3 . Three dimensional $\epsilon_{i j k}$ is defined by $\epsilon_{123}=1$. Roman letters $a, b, c \ldots$ denote the indices of the $\mathrm{SU}(3)$ adjoint representation; they run from 1 to 8 . $f^{a b c}$ are the $\mathrm{SU}(3)$ structure constants and completely antisymmetric with respect to $a, b$, and $c$. Repeated indices of every type are summed over.
} 
and $\bar{\theta} \equiv\left(g^{2} / 8 \pi^{2}\right) \theta$. The hamiltonian is then given by

$$
\tilde{H} \equiv \int d \vec{x}\left(\pi_{i}^{a} \dot{G}_{i}^{a}-\mathcal{L}\right)=\int d \vec{x}\left[\frac{1}{2}\left(\pi_{i}^{a}+\bar{\theta} B_{i}^{a}\right)^{2}+\frac{1}{2} B_{i}^{a 2}+\pi_{i}^{a}\left(\mathcal{D}_{i} G_{0}^{a}\right)\right]
$$

where the tilde is used to distinguish $\tilde{H}$ from its gauge invariant counterpart $H$; see equations below.

The equations of motion for the canonical fields are the Heisenberg equations

$$
\begin{aligned}
\dot{G}_{i}^{a}(x) & =i\left[\tilde{H}, G_{i}^{a}(x)\right] \\
\dot{\pi}_{i}^{a}(x) & =i\left[\tilde{H}, \pi_{i}^{a}(x)\right]
\end{aligned}
$$

in terms of the equal time commutation relations. The hamiltonian has an explicit $x^{0}$-dependence through $G_{0}^{a}$. $\tilde{H}$ at the same $x^{0}$ is used for determining the time evolution of $G_{i}^{a}(x)$ and $\pi_{i}^{a}(x)$ in (8) and (9). [Throughout this paper, our commutator is taken only at equal time. Thus two operators in commutators are understood to have the same time argument if they have $x^{0}$-dependence.] We give initial operator configurations for the canonical fields at $x^{0}=0$ such that they obey the commutators

$$
\begin{aligned}
{\left[\pi_{i}^{a}(\vec{x}), G_{j}^{b}(\vec{y})\right] } & =-i \delta_{i j} \delta^{a b} \delta(\vec{x}-\vec{y}) \\
{\left[\pi_{i}^{a}(\vec{x}), \pi_{j}^{b}(\vec{y})\right] } & =\left[G_{i}^{a}(\vec{x}), G_{j}^{b}(\vec{y})\right]=0 .
\end{aligned}
$$

The equations (8) and (9) determine the canonical fields for all $x$ and the solutions $G_{i}^{a}(x)$ and $\pi_{i}^{a}(x)$ obey (10) and (11) for any $x^{0}$.

It is convenient to define the operator fields $E_{i}^{a}$, chromo-electric field, by

$$
E_{i}^{a}(x) \equiv \pi_{i}^{a}(x)+\bar{\theta} B_{i}^{a}(x)
$$

and to write commutators and Heisenberg equations in terms of $G_{i}^{a}, E_{i}^{a}$, and $B_{i}^{a}$. Using (10)-(12) we obtain

$$
\left[G_{i}^{a}(\vec{x}), G_{j}^{b}(\vec{y})\right]=\left[B_{i}^{a}(\vec{x}), G_{j}^{b}(\vec{y})\right]=\left[B_{i}^{a}(\vec{x}), B_{j}^{b}(\vec{y})\right]=0,
$$




$$
\begin{aligned}
& {\left[E_{i}^{a}(\vec{x}), G_{j}^{b}(\vec{y})\right]=-i \delta_{i j} \delta^{a b} \delta(\vec{x}-\vec{y})} \\
& {\left[E_{i}^{a}(\vec{x}), B_{j}^{b}(\vec{y})\right]=i \epsilon_{i j k}\left\{\delta^{a b} \partial_{k} \delta(\vec{x}-\vec{y})-g f^{a b c} G_{k}^{c}(\vec{y}) \delta(\vec{x}-\vec{y})\right\}} \\
& {\left[E_{i}^{a}(\vec{x}), E_{j}^{b}(\vec{y})\right]=0}
\end{aligned}
$$

while the hamiltonian becomes

$$
\tilde{H}=\int d \vec{x}\left\{\frac{1}{2} E_{i}^{a 2}+\frac{1}{2} B_{i}^{a 2}-\left(\mathcal{D}_{i} E_{i}^{a}\right) G_{0}^{a}\right\} .
$$

Here we have used the equations

$$
\mathcal{D}_{i} \pi_{i}^{a}=\mathcal{D}_{i} E_{i}^{a}
$$

which are the consequences of Bianchi identities

$$
\epsilon^{\mu \nu \lambda \sigma} \mathcal{D}_{\nu} G_{\lambda \sigma}^{a}=0
$$

for $\mu=0$. We have also neglected a surface integral at spatial infinity in Eq. (17). In this paper we neglect similar surface integrals under the assumption that they cannot change the dynamics. The Heisenberg equations now read

$$
\begin{aligned}
\dot{G}_{i}^{a} & =i\left[\tilde{H}, G_{i}^{a}\right]=E_{i}^{a}+\mathcal{D}_{i} G_{0}^{a} \\
\dot{E}_{i}^{a} & =i\left[\tilde{H}, E_{i}^{a}\right]=-\epsilon_{i j k} \mathcal{D}_{j} B_{k}^{a}-g f^{a b c} G_{0}^{b} E_{i}^{c},
\end{aligned}
$$

and

$$
\dot{B}_{i}^{a}=i\left[\tilde{H}, B_{i}^{a}\right]=\epsilon_{i j k} \mathcal{D}_{j} E_{k}^{a}-g f^{a b c} G_{0}^{b} B_{i}^{c} .
$$

Eqs. (20) clearly mean operator fields $E_{i}^{a}$ defined by (12) can be identified as electric fields $G_{0 i}^{a}$. Eqs. (21) are the operator version of the Yang-Mills equations

$$
\mathcal{D}_{\mu} G^{a \mu \nu}=0
$$

for $\nu=i$. Eqs. (22), which are consistent with (5) and (20), are the spatial components of Bianchi identities (19). 
The time components of the Yang-Mills equations,

$$
\mathcal{G}^{a} \equiv \mathcal{D}_{i} E_{i}^{a}=0
$$

cannot be satisfied as an operator equation. Instead, it should be interpreted as a constraint on the physical states |\rangle$[17,12]$. They are defined by

$$
\mathcal{G}^{a}(\vec{x})|\rangle=0
$$

for all $a$ and $\vec{x}$ with $\mathcal{G}^{a}$ at $x^{0}=0$. Although the time derivative of $\mathcal{G}^{a}$ is not zero,

$$
\dot{\mathcal{G}}^{a}=i\left[\tilde{H}, \mathcal{G}^{a}\right]=-g f^{a b c} G_{0}^{b} \mathcal{G}^{c},
$$

its matrix elements in the physical space are zero. This means (25) holds for arbitrary $x^{0}$.

For the verification of the Poincare invariance, we need one more definition. We refer to the hermitian operators that map the physical states into the physical ones as physical. A physical operator $\mathcal{O}_{\mathrm{ph}}$ must satisfy

$$
\left[\mathcal{G}^{a}(x), \mathcal{O}_{\mathrm{ph}}\right]|\rangle=0
$$

for arbitrary $a, x$ and physical |\rangle . This definition means that matrix representation of $\mathcal{O}_{\mathrm{ph}}$ are block diagonal with respect to the physical space and the unphysical space (the orthogonal complement of the physical space). Let us refer to sub-matrix of $\mathcal{O}_{\mathrm{ph}}$ in the physical space as the physical component.

The operators $\mathcal{G}^{a}$ generate local gauge transformations: they satisfy

$$
\begin{aligned}
{\left[\mathcal{G}^{b}(\vec{x}), G_{i}^{a}(\vec{y})\right] } & =-i\left\{g f^{a b c} G_{i}^{c}(\vec{y}) \delta(\vec{x}-\vec{y})+\delta^{a b} \partial_{i} \delta(\vec{x}-\vec{y})\right\}, \\
{\left[\mathcal{G}^{b}(\vec{x}), E_{i}^{a}(\vec{y})\right] } & =-i g f^{a b c} E_{i}^{c}(\vec{y}) \delta(\vec{x}-\vec{y}), \\
{\left[\mathcal{G}^{b}(\vec{x}), B_{i}^{a}(\vec{y})\right] } & =-i g f^{a b c} B_{i}^{c}(\vec{y}) \delta(\vec{x}-\vec{y}),
\end{aligned}
$$

and local algebra of gauge group

$$
\left[\mathcal{G}^{a}(\vec{x}), \mathcal{G}^{b}(\vec{y})\right]=i g f^{a b c} \mathcal{G}^{c}(\vec{y}) \delta(\vec{x}-\vec{y}) .
$$

Thus the gauge invariant operators, the operators that commute with $\mathcal{G}^{a}$, are physical. 


\section{The Poincare algebra}

Now we will explicitly show the Poincare invariance of our quantization scheme, i.e. we will show the existence of Poincare group representation in the physical space. We start with the classical expression of the energy momentum tensor

$$
\theta^{\mu \nu}=-G^{a \mu \lambda} G_{\lambda}^{a \nu}+\frac{1}{4} g^{\mu \nu} G_{\lambda \sigma}^{a} G^{a \lambda \sigma}
$$

and examine its properties in terms of the field operators. The corresponding operator expressions for $\theta^{\mu \nu}$ are

$$
\begin{aligned}
\theta^{00}(x) & =\frac{1}{2}\left\{E_{i}^{a}(x)^{2}+B_{i}^{a}(x)^{2}\right\} \\
\theta^{0 i}(x) & =-\epsilon_{i j k} \frac{1}{2}\left\{E_{j}^{a}(x) B_{k}^{a}(x)+B_{k}^{a}(x) E_{j}^{a}(x)\right\} \\
\theta^{i j}(x) & =\frac{1}{2} \delta_{i j}\left\{E_{i}^{a}(x)^{2}+B_{i}^{a}(x)^{2}\right\}-E_{i}^{a}(x) E_{j}^{a}(x)-B_{i}^{a}(x) B_{j}^{a}(x),
\end{aligned}
$$

where we have symmetrized the expression for $\theta^{0 i}$ with respect to the order of $E_{j}^{a}$ and $B_{k}^{a}$ because they do not commute as operator fields; we will use the same prescription for handling the order of operators when this becomes a problem. $\theta^{0 i}$ satisfy $\theta^{0 i}=\theta^{i 0}$ and are hermitian. We will prove that the unitary transformations

$$
\Lambda(\lambda, \omega) \equiv \exp \left\{i \lambda_{\mu} P^{\mu}+i \omega_{\mu \nu} M^{\mu \nu}\right\}
$$

defined by the energy-momentum-vector $P^{\mu}$

$$
P^{\mu} \equiv \int d \vec{x} \theta^{0 \mu}(x)
$$

and the angular-momentum-tensor $M^{\mu \nu}$

$$
M^{\mu \nu} \equiv \int d \vec{x}\left(x^{\mu} \theta^{0 \nu}(x)-x^{\nu} \theta^{0 \mu}(x)\right)
$$

with real parameters $\lambda_{\mu}$ and $\omega_{\mu \nu}$, constitute a representation of the Poincare group in the physical space. 
The operators $\theta^{\mu \nu}$ are gauge invariant and, thus, physical. They satisfy divergence equations

$$
\begin{aligned}
\dot{\theta}^{00}+\partial_{j} \theta^{0 j} & =0 \\
\dot{\theta}^{0 i}+\partial_{j} \theta^{j i} & =-E_{i}^{a} \mathcal{G}^{a},
\end{aligned}
$$

which are the simple consequence of (21) and (22). Note that the operators $E_{i}^{a} \mathcal{G}^{a}$ are hermitian and physical: $E_{i}^{a}$ and $\mathcal{G}^{a}$ commute for the same color index a no matter what their spatial coordinates are (See Eq. (29)). And their physical components are zero:

$$
E_{i}^{a} \mathcal{G}^{a}|\rangle=0 \text { for any physical }|\rangle
$$

Thus, although some of $P^{\mu}$ and $M^{\mu \nu}$ (which have $\theta^{0 i}$ in their expressions) are $x^{0}$-dependent, their physical components are $x^{0}$-independent. The unitary transformations $\Lambda(\lambda, \omega)$ then have an unique operation in the physical space no matter what time slice in the Minkowski space we use for evaluating the integrals (37) and (38).

The evaluation of commutators of $\theta^{\mu \nu}$ with respect to the operator fields are straightforward. By Eqs. (13)-(16), we obtain

$$
\begin{aligned}
{\left[\theta^{00}(\vec{x}), G_{i}^{a}(\vec{y})\right]=} & -i E_{i}^{a}(\vec{y}) \delta(\vec{x}-\vec{y}) \\
{\left[\theta^{0 k}(\vec{x}), G_{i}^{a}(\vec{y})\right]=} & i \epsilon_{i j k} B_{j}^{a}(\vec{y}) \delta(\vec{x}-\vec{y}) \\
{\left[\theta^{00}(\vec{x}), E_{i}^{a}(\vec{y})\right]=} & i \epsilon_{i j k}\left\{\left(\mathcal{D}_{j} B_{k}^{a}(\vec{y})\right) \delta(\vec{x}-\vec{y})-B_{k}^{a}(\vec{y}) \partial_{j} \delta(\vec{x}-\vec{y})\right\} \\
{\left[\theta^{0 k}(\vec{x}), E_{i}^{a}(\vec{y})\right]=} & i\left(\delta_{k m} \delta_{i l}-\delta_{k i} \delta_{l m}\right) \\
& \times\left\{\left(\mathcal{D}_{m} E_{l}^{a}(\vec{y})\right) \delta(\vec{x}-\vec{y})-E_{l}^{a}(\vec{y}) \partial_{m} \delta(\vec{x}-\vec{y})\right\} \\
{\left[\theta^{00}(\vec{x}), B_{i}^{a}(\vec{y})\right]=} & -i \epsilon_{i j k}\left\{\left(\mathcal{D}_{j} E_{k}^{a}(\vec{y})\right) \delta(\vec{x}-\vec{y})-E_{k}^{a}(\vec{y}) \partial_{j} \delta(\vec{x}-\vec{y})\right\}
\end{aligned}
$$




$$
\begin{aligned}
{\left[\theta^{0 k}(\vec{x}), B_{i}^{a}(\vec{y})\right]=} & i\left(\delta_{k j} \delta_{i l}-\delta_{k i} \delta_{l j}\right) \\
& \times\left\{\left(\mathcal{D}_{j} B_{l}^{a}(\vec{y})\right) \delta(\vec{x}-\vec{y})-B_{l}^{a}(\vec{y}) \partial_{j} \delta(\vec{x}-\vec{y})\right\} .
\end{aligned}
$$

Using these results, we can calculate the commutators of $P^{\mu}$. But these are not the commutators the generators of translations should obey; for example, from (43) we get

$$
\left[P^{k}, G_{i}^{a}(\vec{x})\right]=-i \partial^{k} G_{i}^{a}-i \mathcal{D}_{i} G_{k}^{a}
$$

which has the residual second term on the right hand side.

A careful inspection on Eqs. (42)-(47), however, tells us a simple modification on $\theta^{0 \mu}$ gives us a right form for the generators of translations. We define

$$
\tilde{\theta}^{0 \mu}=\theta^{0 \mu}-\tilde{\mathcal{G}}^{\mu}
$$

with hermitian $\tilde{\mathcal{G}}^{\mu}$,

$$
\tilde{\mathcal{G}}^{\mu}(x) \equiv \frac{1}{2}\left\{\mathcal{G}^{a}(x) G^{a \mu}(x)+G^{a \mu}(x) \mathcal{G}^{a}(x)\right\} .
$$

The commutators of $\tilde{\theta}^{0 \mu}$ are then

$$
\begin{aligned}
{\left[\tilde{\theta}^{00}(\vec{x}), G_{i}^{a}(\vec{y})\right]=} & -i\left\{E_{i}^{a}(\vec{y})+\mathcal{D}_{i} G_{0}^{a}(\vec{y})\right\} \delta(\vec{x}-\vec{y})+i G_{0}^{a}(\vec{y}) \partial_{i} \delta(\vec{x}-\vec{y}), \\
{\left[\tilde{\theta}^{0 k}(\vec{x}), G_{i}^{a}(\vec{y})\right]=} & i\left(\partial_{k} G_{i}^{a}(\vec{y})\right) \delta(\vec{x}-\vec{y})-i G_{k}^{a}(\vec{y}) \partial_{i} \delta(\vec{x}-\vec{y}), \\
{\left[\tilde{\theta}^{00}(\vec{x}), E_{i}^{a}(\vec{y})\right]=} & i \epsilon_{i j k}\left\{\left(\mathcal{D}_{j} B_{k}^{a}(\vec{y})\right) \delta(\vec{x}-\vec{y})-B_{k}^{a}(\vec{y}) \partial_{j} \delta(\vec{x}-\vec{y})\right\} \\
& +i g f^{a b c} G_{0}^{b}(\vec{y}) E_{i}^{c}(\vec{y}) \delta(\vec{x}-\vec{y}), \\
{\left[\tilde{\theta}^{0 k}(\vec{x}), E_{i}^{a}(\vec{y})\right]=} & i\left(\partial_{k} E_{i}^{a}(\vec{y})\right) \delta(\vec{x}-\vec{y}) \\
& -i\left(\delta_{k m} \delta_{i l}-\delta_{k i} \delta_{l m}\right) E_{l}^{a}(\vec{y}) \partial_{m} \delta(\vec{x}-\vec{y}), \\
{\left[\tilde{\theta}^{00}(\vec{x}), B_{i}^{a}(\vec{y})\right]=} & -i \epsilon_{i j k}\left\{\left(\mathcal{D}_{j} E_{k}^{a}(\vec{y})\right) \delta(\vec{x}-\vec{y})-E_{k}^{a}(\vec{y}) \partial_{j} \delta(\vec{x}-\vec{y})\right\} \\
& +i g f^{a b c} G_{0}^{b}(\vec{y}) B_{i}^{c}(\vec{y}) \delta(\vec{x}-\vec{y}), \\
{\left[\tilde{\theta}^{0 k}(\vec{x}), B_{i}^{a}(\vec{y})\right]=} & i\left(\partial_{k} B_{i}^{a}(\vec{y})\right) \delta(\vec{x}-\vec{y}) \\
& -i\left(\delta_{k j} \delta_{i l}-\delta_{k i} \delta_{l j}\right) B_{l}^{a}(\vec{y}) \partial_{j} \delta(\vec{x}-\vec{y}) .
\end{aligned}
$$


Thus the operators $\tilde{P}^{\mu}$, defined in similar way to (37) with $\tilde{\theta}^{0 \mu}$, satisfy

$$
\begin{aligned}
& {\left[\tilde{P}^{\mu}, G_{i}^{a}(x)\right]=-i \partial^{\mu} G_{i}^{a}(x),} \\
& {\left[\tilde{P}^{\mu}, E_{i}^{a}(x)\right]=-i \partial^{\mu} E_{i}^{a}(x),} \\
& {\left[\tilde{P}^{\mu}, B_{i}^{a}(x)\right]=-i \partial^{\mu} B_{i}^{a}(x) .}
\end{aligned}
$$

Since $\tilde{P}^{\mu}$ are not $x^{0}$-independent, these relations does not necessarily mean $\tilde{P}^{\mu}$ are the generators of finite translations. But at least they generate infinitesimal translations for all operator fields. Note that $\tilde{P}^{0}=\tilde{H}$ so that Eqs. (57)-(59) for $\mu=0$ are the Heisenberg equations (20)-(22).

It is interesting to notice that $\tilde{M}^{\mu \nu}$, defined by (38) with $\theta^{\mu \nu}$ replaced by $\tilde{\theta}^{\mu \nu}$, also satisfy the commutation relations of the generators for infinitesimal Lorentz transformations. Indeed, we obtain

$$
\begin{aligned}
& {\left[\tilde{M}^{\mu \nu}, G_{i}^{a}(x)\right]=-i \Delta^{[\mu \nu]} G_{i}^{a}(x),} \\
& {\left[\tilde{M}^{\mu \nu}, E_{i}^{a}(x)\right]=-i \Delta^{[\mu \nu]} E_{i}^{a}(x),} \\
& {\left[\tilde{M}^{\mu \nu}, B_{i}^{a}(x)\right]=-i \Delta^{[\mu \nu]} B_{i}^{a}(x),}
\end{aligned}
$$

where we have introduced a compact notation $\Delta^{[\alpha \beta]}$ for infinitesimal Lorentz transformations. They stand for

$$
\Delta^{[\alpha \beta]} G_{\mu}^{a}=\chi_{\mu \nu}^{[\alpha \beta]} G^{a \nu}+\chi_{\lambda \sigma}^{[\alpha \beta]} x^{\lambda} \partial^{\sigma} G_{\mu}^{a},
$$

for the vector potential and

$$
\Delta^{[\alpha \beta]} G_{\mu \nu}^{a}=\chi_{\mu \lambda}^{[\alpha \beta]} G^{a \lambda}{ }_{\nu}+\chi_{\nu \lambda}^{[\alpha \beta]} G^{a}{ }_{\mu}{ }^{\lambda}+\chi_{\lambda \sigma}^{[\alpha \beta]} x^{\lambda} \partial^{\sigma} G_{\mu \nu}^{a}
$$

for the field strength; $\chi_{\mu \nu}^{[\alpha \beta]}$ is the anti-symmetric parameter for the $[\alpha \beta]$ Lorentz transformations,

$$
\chi_{\mu \nu}^{[\alpha \beta]}=\delta_{\mu}^{\alpha} \delta_{\nu}^{\beta}-\delta_{\nu}^{\alpha} \delta_{\mu}^{\beta} .
$$

Note further that although $\tilde{\mathcal{G}}^{\mu}$ are not gauge invariant, they are physical:

$$
\left[\mathcal{G}^{a}(x), \tilde{\mathcal{G}}^{k}(y)\right]=i \mathcal{G}^{a}(y) \partial_{k} \delta(\vec{x}-\vec{y}) .
$$


Thus $\tilde{P}^{\mu}$ and $\tilde{M}^{\mu \nu}$ are also physical.

Now we will prove that physical components of $\tilde{P}^{\mu}$ and $\tilde{M}^{\mu \nu}$ are the same as those of $P^{\mu}$ and $M^{\mu \nu}$, respectively. We assume that there is at least one vacuum state $|0\rangle$ in the physical space and that it is invariant under spatial translations and rotations generated by $\tilde{P}^{i}$ and $\tilde{M}^{j k}$ evaluated at $x^{0}=0$. Note that finite transformations

$$
\tilde{\Lambda}(\lambda, \omega)=\exp \left\{i \lambda_{i} \tilde{P}^{i}+i \omega_{j k} \tilde{M}^{j k}\right\}
$$

do not change the time coordinate when operating on the field operators. Thus the infinitesimal form that $\tilde{P}^{i}$ and $\tilde{M}^{j k}$ satisfy, (57)-(62), are sufficient to prove that $\tilde{\Lambda}$ represent three dimensional translation-rotation group. Under the above assumption we will prove

$$
\tilde{\mathcal{G}}^{\mu}(x)|\rangle=0
$$

for arbitrary physical |\rangle and $x$.

For $\mu=0$, (68) is obvious: since $G_{0}^{a}$ is c-number function, the definitions (25) for physical states and (50) for $\tilde{\mathcal{G}}^{0}$ result in it. For $\mu=i$, the time derivative of $\tilde{\mathcal{G}}^{i}$,

$$
\dot{\tilde{\mathcal{G}}}^{i}=-\left(E_{i}^{a}+\partial_{i} G_{0}^{a}\right) \mathcal{G}^{a},
$$

has zero physical components:

$$
\dot{\tilde{\mathcal{G}}}^{i}|\rangle=0
$$

Thus we only need to prove (68) at $x^{0}=0$. Let us write $\tilde{\mathcal{G}}^{i}$ at $x^{0}=0$ as

$$
\tilde{\mathcal{G}}^{i}(\vec{x})=c^{i}-G_{i}^{a}(\vec{x}) \mathcal{G}^{a}(\vec{x}),
$$

where

$$
c^{i} \equiv-\frac{1}{2}\left[\mathcal{G}^{a}(\vec{x}), G_{i}^{a}(\vec{x})\right]=\left.\frac{8}{2} i \partial_{i} \delta(\vec{x})\right|_{\vec{x}=0} .
$$


Although $c^{i}$ has ill-defined expression and needs an appropriate regularization, it is at most a c-number. Thus we can choose the vacuum $|0\rangle$ in order to determine $c^{i}$ :

$$
\tilde{\mathcal{G}}^{i}(\vec{x})|0\rangle=c^{i}|0\rangle .
$$

Applying $\tilde{\Lambda}$ on both side of this equation and using the assumption that $|0\rangle$ is invariant under $\tilde{\Lambda}$, we get $c^{i}=R^{i}{ }_{j} c^{j}$ for an arbitrary $\mathrm{SO}(3)$ matrix $R_{j}^{i}$; that is, $c^{i}=0$. Thus the physical components of $\tilde{\mathcal{G}}^{\mu}(x)$ are zero and $P^{\mu}$ and $\tilde{P}^{\mu}$ or $M^{\mu \nu}$ and $\tilde{M}^{\mu \nu}$ have the same physical components.

Using (57)-(62) and $H \equiv P^{0}$, we obtain

$$
\begin{aligned}
{[\tilde{H}, H] } & =\left[\tilde{P}^{j}, H\right]=\left[\tilde{P}^{j}, P^{k}\right]=0 \\
{\left[\tilde{M}^{j k}, H\right] } & =0 \\
{\left[\tilde{M}^{j k}, P^{i}\right] } & =i\left(\delta_{i j} P^{k}-\delta_{i k} P^{j}\right) \\
{\left[\tilde{M}^{0 k}, H\right] } & =-i P^{k} \\
{\left[\tilde{M}^{0 k}, P^{i}\right] } & =-i \delta_{i k} H-i \int d \vec{x} x^{k} E_{i}^{a} \mathcal{G}^{a}, \\
{\left[\tilde{M}^{j k}, M^{l m}\right] } & =i\left(\delta_{j l} M^{k m}-\delta_{j m} M^{k l}+\delta_{k m} M^{j l}-\delta_{k l} M^{j m}\right) \\
{\left[\tilde{M}^{0 k}, M^{i j}\right] } & =i\left(\delta_{j k} M^{0 i}-\delta_{i k} M^{0 j}\right)-i \int d \vec{x} x^{k}\left(x^{i} E_{j}^{a} \mathcal{G}^{a}-x^{j} E_{i}^{a} \mathcal{G}^{a}\right), \\
{\left[\tilde{M}^{0 k}, M^{0 i}\right] } & =i M^{i k}-i \int d \vec{x} x^{0} x^{k} E_{i}^{a} \mathcal{G}^{a} .
\end{aligned}
$$

Since the physical operators have no matrix elements between physical states and unphysical states, the relations (74)-(76) are correct for their physical components as well. Recall $E_{i}^{a} \mathcal{G}^{a}$ has zero physical components. Thus the physical components of $M^{\mu \nu}\left(\tilde{M}^{\mu \nu}\right)$ and $P^{\mu}\left(\tilde{P}^{\mu}\right)$ are $x^{0}$-independent阝 and satisfy the Poincare algebra. The operations of the unitary transformations $\Lambda(\lambda, \omega)$ in the physical space are unambiguously determined, and they represent the Poincare group.

\footnotetext{
${ }^{3}$ Although the physical component of $M^{0 k}$ does not commute with that of $\tilde{H}$, the explicit $x^{0}$-dependence in its definition cancel the $x^{0}$-dependence from the commutator in the Heisenberg equations.
} 


\section{Discussions}

Using the fact that physical components of $P^{\mu}$ commute each other, we can obtain basis vectors in physical space as eigen vectors of them. Let us refer this basis as physical basis. The set of physical basis and operator solutions for the operator fields $\pi_{i}^{a}(x)$ (or $\left.E_{i}^{a}(x)\right)$ and $G_{i}^{a}(x)$ are the all we need to know to make physical predictions. Especially, the matrix elements of physical operators with respect to the physical basis are related to gauge invariant physical predictions.

We first discuss about the effect of $G_{0}^{a}$ on physical observables. Since $G_{0}^{a}$ is an arbitrary function of the space time coordinate $x$, it should have introduced violation of the Poincare invariance had it coupled to a physical degree of freedom. Conversely, our manifest construction of the Poincare generators implies that physical predictions are independent from the specific configuration of $G_{0}^{a}$ that we fix when we start the quantization procedure. This statement is assured by the following two things. 1) The physical basis is $G_{0}^{a}$-independent: they are the eigen vectors of the $x^{0}$-independent physical component of $P^{\mu}$ and they are determined at $x^{0}=0$ by the initial configurations of $\pi_{i}^{a}$ and $G_{i}^{a}$ and the parameter $\theta$. 2) The time evolution of the physical components of physical operators $\mathcal{O}_{\mathrm{ph}}$ is $G_{0}^{a}$-independent: $G_{0}^{a}$ can only couple through the Heisenberg equation

$$
\dot{\mathcal{O}}_{\mathrm{ph}}=i\left[\tilde{H}, \mathcal{O}_{\mathrm{ph}}\right]
$$

where they always appear in conjunction with $\mathcal{G}^{a}$, which have zero physical components. Thus the matrix elements of $\mathcal{O}_{\mathrm{ph}}$ with respect to the physical basis are $G_{0}^{a}$-independent.

Now let us turn to the $\theta$-dependence. We notice that all the $\theta$-dependence concentrates in the definition of $E_{i}^{a}$, (12); although we started with the lagrangian with $\theta$-term, there is no explicit $\theta$-dependence in the commutators

(13)-(16), hamiltonian (17), or the Heisenberg equations (20)-(22). Thus 
we will intensively consider the effect of changing $\theta$ in (12). Specifically, we take a following picture for this consideration. We think the canonical fields $\pi_{i}^{a}(\vec{x})$ (at $x^{0}=0$ ) have $\theta$-independent operations in the Hilbert space as the operators that act on the state vectors. The operators $E_{i}^{a}(\vec{x})$, therefore, have $\theta$-dependent operations. (See figure 1 where we represent $E_{i}^{a}$ at different values of $\theta$ by arrows as they map a state vector to another one.) The physical space does not change for all values of $\theta$ : because of Eq. (18), all the constraints on the physical space for different $\theta$ are identically $\mathcal{D}_{i} \pi_{i}^{a}|\rangle=0$. Now our problem is how physical predictions depend on the $\theta$-dependent initial operator configurations $E_{i}^{a}(\vec{x})$ while the physical space is $\theta$-independent and the equations of motion do not have explicit $\theta$-dependence.

The key for answering this question is a transformation

$$
T(\varphi) \equiv e^{i \varphi q}
$$

defined by the so-called topological charge

$$
\left.q \equiv \int d \vec{x} K^{0}(x)\right|_{x^{0}=0}
$$

and real parameter $\varphi$. Here $K^{0}$ is the time component of the current

$$
K^{\mu}=\frac{g^{2}}{32 \pi^{2}} \epsilon^{\mu \nu \lambda \sigma}\left(G_{\nu}^{a} G_{\lambda \sigma}^{a}-\frac{g}{3} f^{a b c} G_{\nu}^{a} G_{\lambda}^{b} G_{\sigma}^{c}\right)
$$

whose divergence is the Pontryagin density $\partial_{\mu} K^{\mu}=\left(g^{2} / 32 \pi^{2}\right) G_{\mu \nu}^{a} \tilde{G}^{a \mu \nu}$. [This divergence equation holds even for the operator fields by simply setting $G_{0 i}^{a}=$ $E_{i}^{a}$ and using (20).] $T(\varphi)$ transforms the operator fields (at $x^{0}=0$ ) as

$$
\begin{aligned}
T(\varphi) E_{i}^{a}(\vec{x}) T(\varphi)^{-1} & =E_{i}^{a}(\vec{x})+\frac{g^{2}}{8 \pi^{2}} \varphi B_{i}^{a}(\vec{x}), \\
T(\varphi) G_{i}^{a}(\vec{x}) T(\varphi)^{-1} & =G_{i}^{a}(\vec{x})
\end{aligned}
$$

i.e., it connects the initial operator configurations at $\theta$ with those at $\theta+\varphi$. Let $\mathcal{O}_{\theta}(x)$ denote collectively the operator solutions, $E_{i}^{a}(x)$ and $G_{i}^{a}(x)$, for 
the Heisenberg equations with the initial operator configurations at $\theta$. Then define

$$
\mathcal{O}_{\theta+\varphi}(x)=T(\varphi) \mathcal{O}_{\theta}(x) T(\varphi)^{-1}
$$

$\mathcal{O}_{\theta+\varphi}(x)$ also satisfies the equations of motion (20)-(21) and has the appropriate initial configurations for $\theta+\varphi$. [Note that time evolution of $\mathcal{O}_{\theta+\varphi}$ is governed by the hamiltonian written in terms of $\mathcal{O}_{\theta+\varphi}$ themselves.] Thus the operator fields at different values of $\theta$ are related by (83).

Next, we consider the effect of changing $\theta$ on the physical basis. The operators $P^{\mu}$ and $M^{\mu \nu}$ are written in terms of $E_{i}^{a}$ (and $B_{i}^{a}$ ) and, thus, they also have $\theta$-dependent operations. Especially different values of $\theta$ lead us to different representations of the Poincare group and different sets of physical basis. Obviously, $P^{\mu}$ or $M^{\mu \nu}$ at $\theta$ and $\theta+\varphi$ are related by

$$
\begin{gathered}
P_{\theta+\varphi}^{\mu}=T(\varphi) P_{\theta}^{\mu} T(\varphi)^{-1}, \\
M_{\theta+\varphi}^{\mu \nu}=T(\varphi) M_{\theta}^{\mu \nu} T(\varphi)^{-1} .
\end{gathered}
$$

Since $q$ is physical,

$$
\left[\mathcal{G}^{a}(\vec{x}), q\right]=i \frac{g^{2}}{32 \pi^{2}} \int d \vec{y} \epsilon_{i j k}\left(\partial_{j} G_{k}^{a}(\vec{y})\right) \partial_{i} \delta(\vec{x}-\vec{y})=0,
$$

the transformation $T(\varphi)$ is unitary within the physical space. Thus physical basis at $\theta+\varphi,|\rangle_{\theta+\varphi}$, is related to one at $\theta,|\rangle_{\theta}$, by

$$
|\rangle_{\theta+\varphi}=T(\varphi)|\rangle_{\theta}
$$

Eqs. (83) and (87) assert that two theories with different values of $\theta$ yield the same physical predictions and have the same physical content.

The possibility of encountering a physical quantity with a nontrivial $\theta$ dependence can only occur if we can further restrict the physical space into a smaller subspace where the transformation $T(\varphi)$ loses its unitarity. By the requirement of Poincare invariance, this restricted space must be large 
enough to retain the Poincare algebra generated by $P^{\mu}$ and $M^{\mu \nu}$. Usually assumed in the literature [3, 4, 5, 6, 7] is the existence of the "large" gauge transformation $\Omega$ that transforms the operator fields as

$$
\begin{aligned}
t^{a} \Omega G_{i}^{a}(\vec{x}) \Omega^{-1} & =h(\vec{x})^{-1} t^{a} h(\vec{x}) G_{i}^{a}(\vec{x})+\frac{i}{g} h(\vec{x})^{-1} \partial_{i} h(\vec{x}), \\
t^{a} \Omega E_{i}^{a}(\vec{x}) \Omega^{-1} & =h(\vec{x})^{-1} t^{a} h(\vec{x}) E_{i}^{a}(\vec{x})
\end{aligned}
$$

where $t^{a}$ is the $3 \times 3$ hermitian traceless generators for $\mathrm{SU}(3)$ and $h(\vec{x})$ denotes a representative of local gauge transformation which has unit winding number as a map $\mathrm{S}^{3} \rightarrow \mathrm{SU}(3)$. Once we have the explicit $\Omega$, we can further restrict the physical space by the requirement

$$
\Omega|\rangle=|\rangle \text {. }
$$

Since $\Omega$ commutes with $P^{\mu}$ and $M^{\mu \nu}$, the Poincare group is representable within the physical space further restricted by (90). But $\Omega$ does not commute with $q$ and, thus, $T(\varphi)$ is no longer a unitary transformation in this restricted physical space.

$\Omega$ cannot be obtained simply by accumulating infinitesimal gauge transformations generated by $\mathcal{G}^{a}$. Rather, the reason why QCD may have a nontrivial $\theta$-dependence is that the large gauge transformation is disconnected from those generated by $\mathcal{G}^{a}$ [7, 13]. As far as we know, a satisfactory operator expression for $\Omega$ have not been given yet. It is important to construct $\Omega$ explicitly to verify the multi vacua speculation and further examine the $\theta$-dependence of QCD.

\section{Acknowledgement}

The author thanks J. Wudka for helpful discussions. This work is in part supported by the US Department of Energy under Contract No. DE-AT0387ER40327. 


\section{References}

[1] A. Belavin, A. Polyakov, A. Schwartz and Y. Tyupkin, Phys. Lett. 59B (1975) 85.

[2] G. 't Hooft, Phys. Rev. Lett. 37 (1976) 8; Phys. Rev. D14 (1976) 3432.

[3] R. Jackiw and C. Rebbi, Phys. Rev. Lett. 37 (1976) 172;

[4] C. Callan, R. Dashen and D. Gross, Phys. Lett. 63B (1976) 334.

[5] R. Jackiw, Rev. Mod. Phys. 49 (1977) 681.

[6] C. Callan, R. Dashen and D. Gross, Phys. Rev. D17 (1978) 2717.

[7] R. Jackiw, Rev. Mod. Phys. 52 (1980) 661.

[8] V. N. Gribov, Nucl. Phys. B139 (1978) 1, I. Singer, Comm. Math. Phys. 60 (1978) 7.

[9] J. Friedman and N. Papastamatiou, Nucl. Phys. B219 (1983) 125.

[10] I. Bars and F. Green, Nucl. Phys. B142 (1978) 157.

[11] H. Kikuchi and J. Wudka, University of California, Riverside preprint UCRHEP-T101.

[12] H. Weyl, Theory of Groups \& Quantum Mechanics, Chapter IV, Section 12, (Dover,1950).

[13] Y. Wu and A. Zee, Nucl. Phys. B258 (1985) 157 